\title{
Associations of Small Business Closure and Reduced Urban Mobility with Mental Health Problems in COVID-19 Pandemic: a National Representative Sample Study
}

\author{
JungHo Park • Byoungjun Kim $\mathbb{D}$
}

Accepted: 17 December 2020 / Published online: 8 January 2021

(C) The New York Academy of Medicine 2021

\begin{abstract}
It is suggested that the nationwide social distancing due to coronavirus disease 2019 (COVID-19) has adverse mental health consequences despite its necessity. We investigated the associations of social distancing measures with mental health problems. Using national representative sample of 509,062 adults in the USA, we examined the associations of small business closure and reduced urban mobility with generalized anxiety disorder (GAD) and major depression disorder (MDD). Multilevel regression models were fitted with individual, household, and state-level covariates, in addition to state and censusregion-level random effects. Living in state with the highest quartile of small business closures was associated with increased prevalence of GAD (OR: 1.06; CI: 1.031.11) compared to lowest quartile, but had no association with MDD. Living in the highest quartile of urban mobility was associated with lower prevalence of both GAD (OR: 0.88; CI: 0.85-0.93) and MDD (OR: 0.90; CI: $0.86-0.95)$ relative to the lowest quartile. Our findings suggest that small business closures and reduced mobility during COVID-19 pandemic were negatively associated with the two mental health outcomes in the USA, despite their important roles in preventing the infection.
\end{abstract}

J. Park

Sol Price School of Public Policy, University of Southern California, Los Angeles, CA, USA

B. $\operatorname{Kim}(\bowtie)$

Department of Epidemiology, Columbia University Mailman School of Public Health, New York, NY, USA

e-mail: bk2767@cumc.columbia.edu
Keywords COVID-19 pandemic - Generalized anxiety disorder (GAD) • Major depression disorder (MDD) . Small business closure - Urban mobility

\section{Introduction}

During the coronavirus disease 2019 (COVID-19) pandemic, physical and social distancing measures have been implemented across states in the USA to prevent the transmission of disease. These statewide and local interventions range from mandatory quarantine to voluntary self-isolation and have come at a cost of isolating citizens from social activities, potentially increasing risks of development, and/or aggravation of mental health problems [1-5]. Recent surveys of national representative samples showed the adverse mental health consequences during COVID-19 lockdown [6, 7]. For example, in mid-June 2020 , prevalence of anxiety $(25.5 \%)$ and depression (24.3\%) was substantially higher than national estimates from the 2019 US National Health Interview Survey (6.6\% for anxiety and $8.2 \%$ for depression) $[6,8]$. Also, the rates of anxiety or depression during COVID-19 showed clear gradient and even worsening disparity across different demographic and socioeconomic groups, including a higher level of the mental disorders linked with younger age, female, race/ethnicity other than nonHispanic white and nonHispanic Asian, lower education, and lower household income $[6,8]$.

In addition to the individual-level factors, supraindividual predictors, such as urban and spatial contexts, may play important roles in the mental health 
consequences of COVID-19 [9, 10]. Business districts and socializing places have been affected by the closure of numerous small businesses such as restaurants, pubs, and sports facilities, resulting in sharp reductions of routine going-out and changes in behavioral patterns in the USA. [3] A national survey suggested that $41.4 \%$ of small businesses experienced temporary closures due to COVID-19 at least 1 day in late April [11]. For the same period, daily going-out was $32.0 \%$ lower than prepandemic normal days $[12,13]$. Despite the public health crisis, however, the impacts of such contextual and behavioral changes on mental health problems still remain unclear [9]. Thus, we hypothesize that, during COVID-19 pandemic, states with a higher closure rate of small businesses and a lower level of urban mobility may experience increased prevalence rates of mental health outcomes, measured as generalized anxiety disorder (GAD) and major depression disorder (MDD).

\section{Methods}

Data

Main data is the Household Pulse Survey (HPS), a national representative survey deployed by the US Census Bureau jointly with the US National Center for Health Statistics (NCHS), which measures health impacts of COVID-19 pandemic on adult in the USA [14] The HPS comprises with three phases in the year of 2020 : phase 1 (12 surveys during April 23rd-July 21st), phase 2 (5 surveys during August 19th-Oct 26th), and phase 3 (4 surveys during Oct 28th-December 21st). Due to data availability at the time of the analysis, we used the Public Use Data File of the phase 1 of HPS which contains the 12 surveys.

The structure of HPS dataset has a unique property, as it is a combination of longitudinal and cross-sectional compositions. Upon participants' consents, the US Census Bureau collected responses from the same participants up to three times, to achieve the sample size required for a valid national representative survey design [14]. Thus, some of the respondents participated more than once-for either two or three consecutive weeks (longitudinal composition)—while other participants were single-time respondents (cross-sectional composition). The multiple-time respondents (i.e., more than one participation) accounted for a relatively small portion of the total responses - among all participants of the phase 1 survey, $20.6 \%$ responded twice, and $7.1 \%$ responded three times (see Supplemental Table 1 for count of responses by week and response round). Due to the complex survey design, several different analytic approaches have been proposed to address the data structure, but the results were not substantially different from each other [15].

For this analysis, we addressed this unique data structure by dropping the second or third-time responses of the multiple-time respondents and built a pooled cross-sectional dataset that consists of 398,413 firsttime responses (79.4\% of all responses). This approach allowed large sample sizes across states and survey waves. As one of sensitivity tests, we build a separate longitudinal sample that consists of 24,361 respondents who interviewed fully three times and test whether the longitudinal data suggest the same or different results.

\section{Statistical Analysis}

We performed all analyses with Stata/MP 13.1 software (StataCorp, College Station, TX). We conducted separate analyses for GAD and MDD. Multilevel modeling was employed with adult individual and household-level characteristics at level-1, states at level-2, and census regions at level-3. We used multilevel mixed-effects logistic regression models (melogit in Stata/MP 13.1) to estimate the associations of small business closures and reduced mobility with GAD and MDD in a random intercept model, with adjustment for individual and household-level variables and state-level COVID-19 conditions (For a detailed discussion of multilevel mixedeffects logistic models, see Raudenbush and Bryk, 2002 [16], and StataCorp, 2019 [17]).

\section{Mental Health Outcomes}

Two self-reported measures of GAD and MDD were utilized: PHQ-2 ${ }^{18}$ and GAD-2 [18]. The two questionnaire measures frequencies of depression and anxiety symptoms over the last 7 days. The stem question of both PHQ-2 and GAD-2 is "over the last two weeks, how often have you been bothered by any of the following problems?", and the two items of PHQ-2 are "little interest or pleasure in doing things" and "feeling, down, depressed, or hopeless"; and the questions of GAD-2 are "feeling nervous, anxious or on edge" and "not being able to stop or control worrying". For each item, the answers were assigned numeric values: not at all $=0$, 
several days $=1$, more than half the days $=2$, and nearly every day $=3$, and scores from each questionnaire were summed and classified for binary diagnoses: more than three points from PHQ-2 as MDD and more than three points from GAD-2 as GAD. The cutoff points of the PHQ-2 and GAD-2 has been validated for diagnosed MDD and GAD [11, 18, 19].

\section{Statewide Small Business Closure and Mobility} Measures

We used SBPS - another new and nationally representative survey administered by the US Census Bureauwhich measures the changes in small business (1-499 employees) conditions aggregated for all sectors across the same weeks of HPS [11]. We first define the statelevel closure rate of small businesses as percentage of businesses that temporarily closed any of their locations for at least 1 day in the past week. The continuous measure is then used to specify quartile distribution of 50 states and the District of Columbia on a weekly basis.

Urban mobility was measured by an open-source smartphone data in a similar way as the CDC's COVID-19 Data Tracker does [13]. Descartes Labs, a geospatial data provider, quantifies the level of daily urban mobility aggregated at the state level. We define the rate of urban mobility as percentage of the prepandemic normal level $(100 \%$ in the first week of March 2020) and daily measures are averaged to match individual weeks of HPS. It is utilized to identify quartile distribution on a weekly basis.

\section{Statewide COVID-19 Cases and Reopening Policy}

First, statewide COVID-19 cases per 100 population was retrieved from the CDC's COVID-19 Data Tracker [12]. During our analytic period (April 23-June 30), daily count of new cases in the nation gradually declined from 31,760 cases on April 23 to the lowest of 17,027 cases on June 8 but then sharply increased and added 47,717 cases on June 30 , though the trend and pace varied across states. Daily counts are averaged to match individual weeks of HPS data, and the averaged count is divided by time-invariant population and multiplied by 100 .

Second, we used an Indiana University's open-source data on state reopening policies [20]. The data define individual state's reopening date as the earliest date at which that state issued a reopening policy of any type. By June 1, all states and the District of Columbia had reopened in some form (see Supplemental Table 2 for variations in statewide reopening by state and week). We define state reopening policy as a binary variable which equates one if a respondent resides in state that executed at least the first phase of reopening and zero otherwise.

Individual and Household-Level Covariates

Potential individual and household-level confounding covariates are derived from HPS data, including demographic characteristics, socioeconomic status, self-rated health and health care access, and other COVID-19 hardships. Demographic characteristics consist of age, gender, race/ethnicity, marriage, household size, and number of children. Socioeconomic status includes education, household income, and tenure of residence. To control for health-related covariates, we consider selfrated health and insurance status, in addition to coronavirus-specific variables such as incidence of delay and missing of medical care due to COVID-19.

A wide range of socioeconomic hardships during the pandemic may affect mental health, which were controlled by three individual-level covariates: employment income loss [21], food insufficiency [22], and housing instability [23]. Income loss was measured from respondent answers that he/she or anyone in his/her household experienced a loss of employment income since March 13. Respondent reports that he/she did not have an enough of the kinds of food he/she wanted to eat for the last 7 days. Lastly, housing instability was measured whether respondent replies that he/she did not pay his/ her last month's rent or mortgage on time.

Spatiotemporal Effects

To detect the temporal variations during the analytic period, we included the wave indicator as a continuous covariate starting from 1 to 9 . A further time-invariant fixed-effect added in the model is residency in one of the 15 largest metropolitan statistical areas. We considered the metropolitan fixed-effect to control for additional differences in mental health outcomes across the nation's largest metropolitan areas beyond state-level variations.

\section{Results}

Table 1 shows the prevalence of GAD during COVID19 pandemic increased gradually over weeks from 
Table 1 Descriptive statistics: Household Pulse Survey, April 23-June 30, 2020

\begin{tabular}{|c|c|c|c|c|c|}
\hline \multirow[t]{2}{*}{ Variable } & \multirow{2}{*}{$\begin{array}{l}\text { Full sample } \\
(n=398,413), \\
\% \text { or mean } \pm \mathrm{SD}\end{array}$} & \multicolumn{2}{|c|}{ Generalized anxiety disorder (GAD) } & \multicolumn{2}{|c|}{ Major depression disorder (MDD) } \\
\hline & & $\begin{array}{l}\text { Yes }(n=128,254) \\
\% \text { or mean } \pm \text { SD }\end{array}$ & $\begin{array}{l}\text { No }(n=270,159) \\
\% \text { or mean } \pm \text { SD }\end{array}$ & $\begin{array}{l}\text { Yes }(n=90,327), \\
\% \text { or mean } \pm \text { SD }\end{array}$ & $\begin{array}{l}\text { No }(n=308,086), \\
\% \text { or mean } \pm \text { SD }\end{array}$ \\
\hline
\end{tabular}

Demographic characteristics

Age

$18-24$ (Ref) $\quad 9.5$

25-34

$35-44$

$45-54$

$55-64$

$65+$

Gender

Female (Ref)

Male

Race/ethnicity

NonHispanic White (Ref)

Non-Hispanic Black

NonHispanic A\&PI

NonHispanic other

Hispanic

Marital status

Unmarried (Ref)

Married

Children in household

No child (Ref)

One or more children

Household size

Single person (Ref)

2-person

3-person

4-person

5-person

6 or more persons

Socioeconomic status (SES)

Education

Less than high school (Ref)

High school

Some college and AA

$\mathrm{BA}+$

Household income

Less than $\$ 25,000$ (Ref)

$\$ 25,000-49,999$

$\$ 50,000-74,999$

$\$ 75,000-99,999$

$\$ 100,000-\$ 149,999$
8.9

9.5
25.6

21.0

17.1

14.7

12.0

51.3

48.7

57.5

13.7

5.3

4.0

19.5

47.8

52.2

55.2

44.8

7.2

26.4

20.3

20.5

11.9

13.8

31.4

28.5

16.7

25.4

18.0

13.2

14.4
11.1

29.4

21.4

16.9

12.9

8.3

57.9

42.2

56.7

13.7

4.3

4.8

20.6

54.6

45.4

54.1

45.9

7.0

25.0

20.8

20.9

12.2

14.1

9.6

31.0

34.0

25.4

22.0

27.8

18.1

11.8

11.7
8.6

23.6

20.8

17.2

15.7

14.0

47.8

52.2

58.0

13.7

5.8

3.6

18.9

44.1

55.9

55.8

44.2

7.3

27.2

19.9

20.3

11.7

13.6

8.5

31.2

30.1

30.1

13.9

24.0

18.0

13.9

15.9
13.4

29.3

19.7

15.9

12.9

8.8

55.3

44.7

54.5

14.6

4.7

4.9

21.4

59.4

40.5

56.6

43.4

54.7

45.3

7.7

25.4

20.6

19.8

12.0

14.5

7.0

26.8

20.1

20.8

11.8

13.5

\section{Springer}


Table 1 (continued)

\begin{tabular}{|c|c|c|c|c|c|}
\hline \multirow[t]{2}{*}{ Variable } & \multirow{2}{*}{$\begin{array}{l}\text { Full sample } \\
(n=398,413), \\
\% \text { or mean } \pm \text { SD }\end{array}$} & \multicolumn{2}{|c|}{ Generalized anxiety disorder (GAD) } & \multicolumn{2}{|c|}{ Major depression disorder (MDD) } \\
\hline & & $\begin{array}{l}\text { Yes }(n=128,254) \\
\% \text { or mean } \pm \text { SD }\end{array}$ & $\begin{array}{l}\text { No }(n=270,159) \text {, } \\
\% \text { or mean } \pm \text { SD }\end{array}$ & $\begin{array}{l}\text { Yes }(n=90,327) \\
\% \text { or mean } \pm \text { SD }\end{array}$ & $\begin{array}{l}\text { No }(n=308,086) \text {, } \\
\% \text { or mean } \pm \text { SD }\end{array}$ \\
\hline$\$ 150,000$ and above & 12.3 & 8.6 & 14.3 & 6.7 & 14.4 \\
\hline \multicolumn{6}{|l|}{ Tenure of residence } \\
\hline Rental housing unit (Ref) & 43.3 & 50.9 & 39.2 & 53.8 & 39.4 \\
\hline Owner housing unit & 56.7 & 49.1 & 60.8 & 46.2 & 60.6 \\
\hline \multicolumn{6}{|l|}{ Self-rated health and health care access } \\
\hline \multicolumn{6}{|l|}{ Self-rated health } \\
\hline Excellent (Ref) & 19.1 & 11.1 & 23.3 & 9.5 & 22.6 \\
\hline Very good & 33.3 & 26.4 & 36.9 & 24.0 & 36.7 \\
\hline Good & 30.3 & 33.9 & 28.4 & 34.1 & 28.9 \\
\hline Fair & 14.1 & 22.2 & 9.8 & 24.8 & 10.2 \\
\hline Poor & 3.2 & 6.4 & 1.6 & 7.6 & 1.6 \\
\hline \multicolumn{6}{|l|}{ Insurance status } \\
\hline Private (Ref) & 52.7 & 48.8 & 54.8 & 45.9 & 55.2 \\
\hline Public & 14.7 & 16.9 & 13.5 & 18.2 & 13.4 \\
\hline Both private and public & 14.7 & 13.4 & 15.4 & 13.6 & 15.2 \\
\hline Other & 6.1 & 6.2 & 6.0 & 6.5 & 6.0 \\
\hline None & 11.8 & 14.6 & 10.3 & 15.9 & 10.3 \\
\hline \multicolumn{6}{|c|}{ Delayed medical care due to pandemic } \\
\hline Yes (Ref) & 40.5 & 53.6 & 33.4 & 53.4 & 35.7 \\
\hline No & 59.5 & 46.4 & 66.6 & 46.6 & 64.3 \\
\hline \multicolumn{6}{|c|}{ Did not get medical care due to pandemic } \\
\hline Yes (Ref) & 32.9 & 45.9 & 26.0 & 46.7 & 27.8 \\
\hline No & 67.1 & 54.1 & 74.0 & 53.2 & 72.1 \\
\hline \multicolumn{6}{|l|}{ COVID-19 hardship } \\
\hline \multicolumn{6}{|l|}{ Employment income loss } \\
\hline No (Ref) & 48.5 & 37.9 & 54.1 & 37.1 & 52.6 \\
\hline Yes & 51.5 & 62.2 & 45.9 & 62.9 & 47.3 \\
\hline \multicolumn{6}{|l|}{ Food insufficiency } \\
\hline No (Ref) & 56.2 & 39.7 & 65.0 & 36.0 & 63.6 \\
\hline Yes & 43.8 & 60.4 & 35.0 & 64.0 & 36.4 \\
\hline \multicolumn{6}{|l|}{ Housing instability } \\
\hline No (Ref) & 87.4 & 81.5 & 90.6 & 80.2 & 90.1 \\
\hline Yes & 12.6 & 18.5 & 9.4 & 19.8 & 9.9 \\
\hline \multicolumn{6}{|l|}{ Spatiotemporal effects } \\
\hline \multicolumn{6}{|l|}{ MSA ( $\%$ of nonMSA or MSA) } \\
\hline NonMSA (Ref) & 100.0 & 34.5 & 65.5 & 26.6 & 73.4 \\
\hline MSA & 100.0 & $35.6^{* * *}$ & 64.4 & $27.7 * * *$ & 72.3 \\
\hline \multicolumn{6}{|l|}{ Week (\% of weekly sample) } \\
\hline Week 1: April 23-May 5 (Ref) & 100.0 & 32.4 & 67.6 & 24.5 & 75.5 \\
\hline Week 2: May 7-12 & 100.0 & $31.7 * *$ & 68.3 & $25.6^{* * *}$ & 74.4 \\
\hline Week 3: May 14-19 & 100.0 & 29.9 & 70.1 & $25.6^{* *}$ & 74.4 \\
\hline Week 4: May 21-26 & 100.0 & $31.1 *$ & 68.9 & $25.6+$ & 74.4 \\
\hline
\end{tabular}


Table 1 (continued)

\begin{tabular}{|c|c|c|c|c|c|}
\hline \multirow[t]{2}{*}{ Variable } & \multirow{2}{*}{$\begin{array}{l}\text { Full sample } \\
(n=398,413), \\
\% \text { or mean } \pm \mathrm{SD}\end{array}$} & \multicolumn{2}{|c|}{ Generalized anxiety disorder (GAD) } & \multicolumn{2}{|c|}{ Major depression disorder (MDD) } \\
\hline & & $\begin{array}{l}\text { Yes }(n=128,254) \\
\% \text { or mean } \pm \text { SD }\end{array}$ & $\begin{array}{l}\text { No }(n=270,159) \text {, } \\
\% \text { or mean } \pm \text { SD }\end{array}$ & $\begin{array}{l}\text { Yes }(n=90,327) \\
\% \text { or mean } \pm \text { SD }\end{array}$ & $\begin{array}{l}\text { No }(n=308,086), \\
\% \text { or mean } \pm \text { SD }\end{array}$ \\
\hline Week 5: May 28-June 2 & 100.0 & $32.6+$ & 67.4 & $26.8 * * *$ & 73.2 \\
\hline Week 6: June 4-9 & 100.0 & $33.0 *$ & 67.0 & 27.3 & 72.7 \\
\hline Week 7: June 11-16 & 100.0 & $33.6^{* * *}$ & 66.4 & $26.1 * * *$ & 73.9 \\
\hline Week 8: June 18-23 & 100.0 & $33.4 * * *$ & 66.6 & $27.2 * * *$ & 72.8 \\
\hline Week 9: June 25-30 & 100.0 & $35.2 * * *$ & 64.8 & $28.7 * * *$ & 71.3 \\
\hline \multicolumn{6}{|c|}{ Statewide COVID-19 conditions and reopening policy (mean and SD) } \\
\hline$\%$ small business closure & $29.65(11.78)$ & $29.32(11.85)$ & $29.82(11.74)$ & $29.4(11.88)$ & $29.74(11.74)$ \\
\hline$\%$ outdoor mobility & $46.47(23.22)$ & $46.85(23.2)$ & $46.28(23.22)$ & $46.84(23.23)$ & $46.34(23.21)$ \\
\hline State reopening policy & $0.88(0.33)$ & $0.89(0.33)$ & $0.88(0.34)$ & $0.89(0.33)$ & $0.88(0.34)$ \\
\hline COVID-19 cases per 100 people & $0.52(0.49)$ & $0.53(0.49)$ & $0.52(0.48)$ & $0.53(0.49)$ & $0.52(0.48)$ \\
\hline
\end{tabular}

A\&PI Asian and Pacific Islander. MSA metropolitan statistical area. Two-sample $t$ test was used to check statistical significance of meandifferences for MSA and week. $* * * p<0.001, * * p<0.01, * p<0.05,+p<0.10$

$32.4 \%$ in late April (week 1) to $35.2 \%$ in late June (week 9), and the prevalence of MDD also increased from 24.5 to $28.7 \%$ for the same period. A greater share of urban residents living in one of the largest 15 MSAs suffered from GAD (35.6\%) and MDD (27.7\%) than nonMSA residents $(34.5 \%$ and $26.6 \%$ for GAD and MDD, respectively). Two-sample $t$ test results show that, for both GAD and MDD, statistically significant differences exist depending on survey week and location of residence. Also, prevalence of GAD and MDD varied substantially across demographic characteristics, socioeconomic status, self-rate health and health care access, and other COVID-19 hardships.

\section{GAD Model Results}

Table 2 shows the results of multilevel mixed-effects logistic models that estimated associations of small business closure and reduced mobility with GAD adjusting for abovementioned individual, household, and state-level covariates.

Living in state with mass small business closures (highest-quartile state) was associated with increased rate of GAD (OR: 1.07; CI: 1.04-1.12) compared to living in state with least closures (lowest-quartile state). However, this was not a linear relationship as visually displayed in Fig. 1. The column graph shows odds ratio of GAD and MDD on $y$-axis and six categorized closure rates of small business on $x$-axis, with $95 \%$ confidence interval (vertical line at the top of each column) by which we can be $95 \%$ certain that the interval range contains the true mean of the population. Adult Americans who were categorized as living in states with closures of $0-10 \%$ had the lowest odds of GAD. A relatively small increase in small business closure to $10.01-20 \%$ and to $20.01-30 \%$ led to the smallest increase in the odds ratio for GAD; however, the odds ratio (relative to the $20.01-30 \%$ closure) rose more for small business closures of $30.01-40 \%$ and $40.01-50 \%$; beyond $50 \%$, increases in small business closure led to the greatest increase in the odds ratio for GAD.

Urban mobility was negatively associated with the rate of GAD. Living in one of the most mobile (highest quartile) states was associated with decreased prevalence of GAD (OR: 0.88; CI: 0.85-0.93) relative to living in one of the least mobile (lowest quartile) states. As shown in Fig. 2, adults who were categorized as living in states with mobility of $0-20 \%$ had the highest odds of GAD. A relatively small increase in mobility to $20.01-40 \%$ and all the way up to $100 \%$ (= prepandemic normal level of mobility) led to the smallest decrease in the odds ratio for GAD.

Odds ratios estimated for individual-level factors were also shown in Table 2. Most of the level-1 covariates emerge as significant predictors of GAD during the pandemic, mostly consistent with the previous research on pandemic mental disorders. Key demographic and socioeconomic predictors of a higher level of GAD 
Table 2 Multilevel mixed effects logistic model results: US Census Bureau's Household Pulse Survey, April 23-June 30, 2020

\begin{tabular}{|c|c|c|c|c|c|c|}
\hline & \multicolumn{3}{|c|}{ Generalized anxiety disorder (GAD) } & \multicolumn{3}{|c|}{ Major depression disorder (MDD) } \\
\hline & OR & $(95 \% \mathrm{CI})$ & $P$ & OR & $(95 \% \mathrm{CI})$ & $P$ \\
\hline \multicolumn{7}{|c|}{ Statewide COVID-19 conditions and reopening policy } \\
\hline \multicolumn{7}{|l|}{$\%$ small business closure } \\
\hline Bottom Q1 state (Ref) & 1.00 & & & 1.00 & & \\
\hline Q2 state & 1.05 & $(1.02,1.08)$ & 0.001 & 1.01 & $(0.98,1.04)$ & 0.640 \\
\hline Q3 state & 1.07 & $(1.04,1.11)$ & $<0.001$ & 1.00 & $(0.98,1.04)$ & 0.796 \\
\hline Top Q4 state & 1.07 & $(1.04,1.12)$ & 0.001 & 0.99 & $(0.95,1.04)$ & 0.733 \\
\hline \multicolumn{7}{|l|}{$\%$ outdoor mobility } \\
\hline Bottom Q1 state (Ref) & 1.00 & & & 1.00 & & \\
\hline Q2 state & 0.98 & $(0.95,1.01)$ & 0.127 & 0.96 & $(0.94,1)$ & 0.043 \\
\hline Q3 state & 0.91 & $(0.88,0.96)$ & $<0.001$ & 0.92 & $(0.88,0.97)$ & $<0.001$ \\
\hline Top Q4 state & 0.88 & $(0.85,0.93)$ & $<0.001$ & 0.90 & $(0.86,0.95)$ & $<0.001$ \\
\hline State reopening policy & 0.95 & $(0.93,0.99)$ & 0.007 & 1.00 & $(0.97,1.05)$ & 0.940 \\
\hline COVID-19 cases per 100 people & 0.98 & $(0.94,1.03)$ & 0.301 & 1.02 & $(0.97,1.09)$ & 0.458 \\
\hline \multicolumn{7}{|l|}{ Demographic characteristics } \\
\hline \multicolumn{7}{|l|}{ Age } \\
\hline 18-24 (Ref) & 1.00 & & & 1.00 & & \\
\hline $25-34$ & 0.92 & $(0.89,0.97)$ & $<0.001$ & 0.74 & $(0.71,0.78)$ & $<0.001$ \\
\hline $35-44$ & 0.76 & $(0.74,0.8)$ & $<0.001$ & 0.59 & $(0.57,0.62)$ & $<0.001$ \\
\hline $45-54$ & 0.61 & $(0.59,0.65)$ & $<0.001$ & 0.51 & $(0.49,0.54)$ & $<0.001$ \\
\hline $55-64$ & 0.48 & $(0.47,0.51)$ & $<0.001$ & 0.42 & $(0.4,0.44)$ & $<0.001$ \\
\hline $65+$ & 0.33 & $(0.32,0.36)$ & $<0.001$ & 0.31 & $(0.3,0.33)$ & $<0.001$ \\
\hline \multicolumn{7}{|l|}{ Gender } \\
\hline Female (Ref) & 1.00 & & & 1.00 & & \\
\hline Male & 0.71 & $(0.7,0.72)$ & $<0.001$ & 0.91 & $(0.9,0.93)$ & $<0.001$ \\
\hline \multicolumn{7}{|l|}{ Race/ethnicity } \\
\hline NonHispanic White (Ref) & 1.00 & & & 1.00 & & \\
\hline NonHispanic Black & 0.69 & $(0.68,0.72)$ & $<0.001$ & 0.77 & $(0.75,0.8)$ & $<0.001$ \\
\hline NonHispanic A\&PI & 0.69 & $(0.67,0.72)$ & $<0.001$ & 0.94 & $(0.91,0.99)$ & 0.007 \\
\hline NonHispanic other & 0.98 & $(0.95,1.02)$ & 0.303 & 1.04 & $(1.01,1.09)$ & 0.048 \\
\hline Hispanic & 0.86 & $(0.84,0.89)$ & $<0.001$ & 0.89 & $(0.87,0.92)$ & $<0.001$ \\
\hline \multicolumn{7}{|l|}{ Marital status } \\
\hline Unmarried (Ref) & 1.00 & & & 1.00 & & \\
\hline Married & 0.88 & $(0.87,0.9)$ & $<0.001$ & 0.80 & $(0.78,0.82)$ & $<0.001$ \\
\hline \multicolumn{7}{|l|}{ Children in household } \\
\hline No child (Ref) & 1.00 & & & 1.00 & & \\
\hline One or more children & 0.99 & $(0.97,1.02)$ & 0.411 & 0.87 & $(0.85,0.9)$ & $<0.001$ \\
\hline \multicolumn{7}{|l|}{ Household size } \\
\hline Single person (Ref) & 1.00 & & & 1.00 & & \\
\hline 2-person & 1.03 & $(1.01,1.06)$ & 0.009 & 0.93 & $(0.91,0.96)$ & $<0.001$ \\
\hline 3-person & 0.99 & $(0.96,1.03)$ & 0.501 & 0.89 & $(0.86,0.92)$ & $<0.001$ \\
\hline 4-person & 0.95 & $(0.92,0.99)$ & 0.005 & 0.85 & $(0.83,0.89)$ & $<0.001$ \\
\hline 5-person & 0.88 & $(0.85,0.92)$ & $<0.001$ & 0.83 & $(0.8,0.87)$ & $<0.001$ \\
\hline 6 or more persons & 0.81 & $(0.78,0.85)$ & $<0.001$ & 0.78 & $(0.75,0.82)$ & $<0.001$ \\
\hline
\end{tabular}


Table 2 (continued)

\begin{tabular}{|c|c|c|c|c|c|c|}
\hline & \multicolumn{3}{|c|}{ Generalized anxiety disorder (GAD) } & \multicolumn{3}{|c|}{ Major depression disorder (MDD) } \\
\hline & OR & $(95 \% \mathrm{CI})$ & $P$ & OR & $(95 \% \mathrm{CI})$ & $P$ \\
\hline \multicolumn{7}{|l|}{ Socioeconomic status (SES) } \\
\hline \multicolumn{7}{|l|}{ Education } \\
\hline Less than high school (Ref) & 1.00 & & & 1.00 & & \\
\hline High school & 1.05 & $(1,1.11)$ & 0.108 & 1.02 & $(0.97,1.08)$ & 0.533 \\
\hline Some college and AA & 1.15 & $(1.1,1.22)$ & $<0.001$ & 1.04 & $(0.99,1.1)$ & 0.160 \\
\hline $\mathrm{BA}+$ & 1.28 & $(1.22,1.35)$ & $<0.001$ & 0.99 & $(0.94,1.05)$ & 0.808 \\
\hline \multicolumn{7}{|l|}{ Household income } \\
\hline Less than $\$ 25,000$ (Ref) & 1.00 & & & 1.00 & & \\
\hline$\$ 25,000-49,999$ & 0.94 & $(0.92,0.98)$ & $<0.001$ & 0.95 & $(0.93,0.98)$ & $<0.001$ \\
\hline$\$ 50,000-74,999$ & 0.94 & $(0.92,0.98)$ & $<0.001$ & 0.92 & $(0.9,0.96)$ & $<0.001$ \\
\hline$\$ 75,000-99,999$ & 0.91 & $(0.89,0.95)$ & $<0.001$ & 0.86 & $(0.84,0.9)$ & $<0.001$ \\
\hline$\$ 100,000-\$ 149,999$ & 0.88 & $(0.86,0.92)$ & $<0.001$ & 0.80 & $(0.78,0.84)$ & $<0.001$ \\
\hline$\$ 150,000$ and above & 0.89 & $(0.86,0.93)$ & $<0.001$ & 0.75 & $(0.73,0.79)$ & $<0.001$ \\
\hline \multicolumn{7}{|l|}{ Tenure of residence } \\
\hline Rental housing unit (Ref) & 1.00 & & & 1.00 & & \\
\hline Owner housing unit & 0.90 & $(0.89,0.92)$ & $<0.001$ & 0.90 & $(0.89,0.93)$ & $<0.001$ \\
\hline \multicolumn{7}{|c|}{ Self-rated health and health care access } \\
\hline \multicolumn{7}{|c|}{ Self-rated health } \\
\hline Excellent (Ref) & 1.00 & & & 1.00 & & \\
\hline Very good & 1.43 & $(1.41,1.47)$ & $<0.001$ & 1.45 & $(1.42,1.49)$ & $<0.001$ \\
\hline Good & 2.15 & $(2.11,2.21)$ & $<0.001$ & 2.35 & $(2.29,2.42)$ & $<0.001$ \\
\hline Fair & 3.69 & $(3.59,3.8)$ & $<0.001$ & 4.22 & $(4.09,4.36)$ & $<0.001$ \\
\hline Poor & 6.65 & $(6.33,7)$ & $<0.001$ & 7.67 & $(7.3,8.07)$ & $<0.001$ \\
\hline \multicolumn{7}{|l|}{ Insurance status } \\
\hline Private (Ref) & 1.00 & & & 1.00 & & \\
\hline Public & 0.97 & $(0.95,1)$ & 0.019 & 1.03 & $(1.01,1.07)$ & 0.036 \\
\hline Both private and public & 0.90 & $(0.88,0.93)$ & $<0.001$ & 0.93 & $(0.91,0.96)$ & $<0.001$ \\
\hline Other & 0.96 & $(0.93,0.99)$ & 0.011 & 0.97 & $(0.94,1.01)$ & 0.114 \\
\hline None & 1.13 & $(1.1,1.17)$ & $<0.001$ & 1.20 & $(1.17,1.25)$ & $<0.001$ \\
\hline \multicolumn{7}{|c|}{ Delayed medical care due to pandemic } \\
\hline Yes (Ref) & 1.00 & & & 1.00 & & \\
\hline No & 0.66 & $(0.65,0.67)$ & $<0.001$ & 0.73 & $(0.72,0.75)$ & $<0.001$ \\
\hline \multicolumn{7}{|c|}{ Did not get medical care due to pandemic } \\
\hline Yes (Ref) & 1.00 & & & 1.00 & & \\
\hline No & 0.71 & $(0.71,0.73)$ & $<0.001$ & 0.70 & $(0.69,0.72)$ & $<0.001$ \\
\hline \multicolumn{7}{|l|}{ COVID-19 hardship } \\
\hline \multicolumn{7}{|l|}{ Employment income loss } \\
\hline No (Ref) & 1.00 & & & 1.00 & & \\
\hline Yes & 1.48 & $(1.46,1.51)$ & $<0.001$ & 1.37 & $(1.35,1.4)$ & $<0.001$ \\
\hline \multicolumn{7}{|l|}{ Food insufficiency } \\
\hline No (Ref) & 1.00 & & & 1.00 & & \\
\hline Yes & 1.84 & $(1.81,1.87)$ & $<0.001$ & 1.94 & $(1.91,1.98)$ & $<0.001$ \\
\hline
\end{tabular}


Table 2 (continued)

\begin{tabular}{|c|c|c|c|c|c|c|}
\hline & \multicolumn{3}{|c|}{ Generalized anxiety disorder (GAD) } & \multicolumn{3}{|c|}{ Major depression disorder (MDD) } \\
\hline & OR & $(95 \% \mathrm{CI})$ & $P$ & OR & $(95 \% \mathrm{CI})$ & $P$ \\
\hline \multicolumn{7}{|l|}{ Housing instability } \\
\hline No (Ref) & 1.00 & & & 1.00 & & \\
\hline Yes & 1.41 & $(1.38,1.46)$ & $<0.001$ & 1.38 & $(1.35,1.42)$ & $<0.001$ \\
\hline \multicolumn{7}{|l|}{ Spatiotemporal effects } \\
\hline \multicolumn{7}{|c|}{15 largest metropolitan areas } \\
\hline None (Ref) & 1.00 & & & 1.00 & & \\
\hline New York & 1.13 & $(1.06,1.22)$ & 0.001 & 1.10 & $(1.02,1.19)$ & 0.018 \\
\hline Los Angeles & 1.14 & $(1.07,1.22)$ & $<0.001$ & 1.16 & $(1.08,1.25)$ & $<0.001$ \\
\hline Chicago & 1.18 & $(1.1,1.28)$ & $<0.001$ & 1.17 & $(1.07,1.28)$ & 0.001 \\
\hline Dallas & 1.00 & $(0.93,1.08)$ & 0.905 & 1.09 & $(1.01,1.18)$ & 0.045 \\
\hline Houston & 1.03 & $(0.96,1.12)$ & 0.394 & 1.13 & $(1.04,1.23)$ & 0.005 \\
\hline Washington, D.C. & 1.13 & $(1.07,1.2)$ & $<0.001$ & 1.13 & $(1.06,1.21)$ & 0.001 \\
\hline Miami & 1.11 & $(1.03,1.2)$ & 0.007 & 1.09 & $(1.01,1.19)$ & 0.037 \\
\hline Philadelphia & 1.13 & $(1.07,1.21)$ & $<0.001$ & 1.08 & $(1.01,1.16)$ & 0.029 \\
\hline Atlanta & 1.12 & $(1.03,1.22)$ & 0.013 & 1.08 & $(0.98,1.19)$ & 0.133 \\
\hline Phoenix & 1.14 & $(1.05,1.25)$ & 0.003 & 1.19 & $(1.09,1.32)$ & $<0.001$ \\
\hline Boston & 1.03 & $(0.97,1.11)$ & 0.357 & 1.02 & $(0.94,1.1)$ & 0.714 \\
\hline San Francisco & 1.17 & $(1.1,1.26)$ & $<0.001$ & 1.12 & $(1.04,1.22)$ & 0.006 \\
\hline Riverside & 0.98 & $(0.91,1.06)$ & 0.569 & 0.99 & $(0.91,1.08)$ & 0.787 \\
\hline Detroit & 0.95 & $(0.88,1.05)$ & 0.292 & 1.00 & $(0.91,1.11)$ & 0.987 \\
\hline Seattle & 1.13 & $(1.05,1.23)$ & 0.002 & 1.16 & $(1.07,1.27)$ & 0.001 \\
\hline Survey week & 1.02 & $(1.02,1.03)$ & $<0.001$ & 1.00 & $(1,1.01)$ & 0.053 \\
\hline Constant & 0.51 & $(0.48,0.57)$ & $<0.001$ & 0.44 & $(0.4,0.49)$ & $<0.001$ \\
\hline Number of observations & 398,413 & & 398,413 & & & \\
\hline Wald chi-squared & 53,531 & & 51,087 & & & \\
\hline Log likelihood & $-216,922$ & $<0.001$ & $-181,880$ & $<0.001$ & & \\
\hline \multicolumn{7}{|l|}{ Likelihood-ratio test } \\
\hline Chi-squared & 78.46 & $<0.001$ & 104.08 & $<0.001$ & & \\
\hline \multicolumn{7}{|l|}{ Intraclass correlation (ICC) } \\
\hline Census region & 0.0000 & $(0.0000,1.0000)$ & & 0.0003 & $(0.0000,1.0000)$ & \\
\hline State & 0.0009 & $(0.0006,0.0016)$ & & 0.0013 & $(0.0008,0.0025)$ & \\
\hline
\end{tabular}

OR odds ratio; $C I$ confidence interval; $A \& P I$ Asian and Pacific Islander; The sample size was $n=398,413$

include younger age, female, unmarried status, smaller household size, lower household income, and renting versus owning a home. Contrary to expectations, nonHispanic white and a higher level of education were associated with a higher risk of GAD, which is likely due to other socioeconomic covariates such as household income and tenure of residence. A higher risk of GAD was also associated with worse self-rated health status, no insurance in any type, and delayed (or missed) medical care due to the pandemic. A further set of important predictors is socioeconomic hardships during the COVID-19 pandemic, suggesting a higher level of GAD associated with employment income loss (OR: 1.48; CI: 1.46-1.51), food insufficiency (OR: 1.84; CI: 1.81-1.87), and delayed housing payment (OR: 1.41; CI: $1.38-1.46)$.

As for spatiotemporal effects, the results show that urban residents who live in some largest 
Fig. 1 Odds ratio of GAD and MDD among adult Americans during the COVID-19 pandemic, by small business closure rate: US Census Bureau's Household Pulse Survey, April 23-June 30, 2020

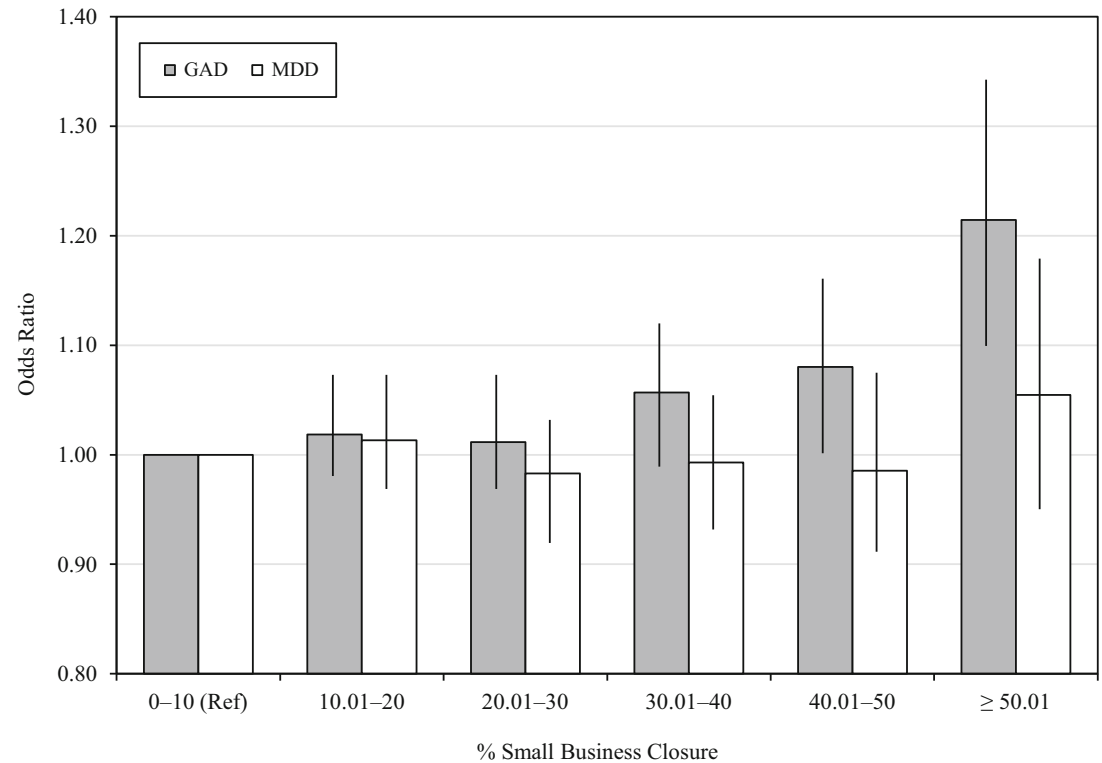

metropolitan areas such as New York and Los Angeles are more likely to suffer from GAD than nonmetropolitan residents. Also, the incidence rate of GAD increases by $2 \%$ per week (OR: 1.02 ; CI: 1.02-1.03) during the analytic period (April 23June 30, 2020) even after controlling for all the other covariates. These findings from the spatiotemporal effects imply that the risk of GAD is higher in the most urbanized areas as the pandemic prolongs over weeks.

\section{MDD Model Results}

Unlike the results of GAD, neither small business closure nor state reopening policy was associated with MDD. However, there was a strong negative association between urban mobility and MDD. Living in one of the most mobile (highest quartile) states was associated with lower prevalence of MDD (OR: 0.90; CI: 0.86-0.95) relative to living in one of the least mobile (highest quartile) states.
Fig. 2 Odds of GAD and MDD among adult Americans during the COVID-19 pandemic, by urban mobility rate relative to the pre-pandemic normal level: US Census Bureau's Household Pulse Survey, April 23-June 30, 2020

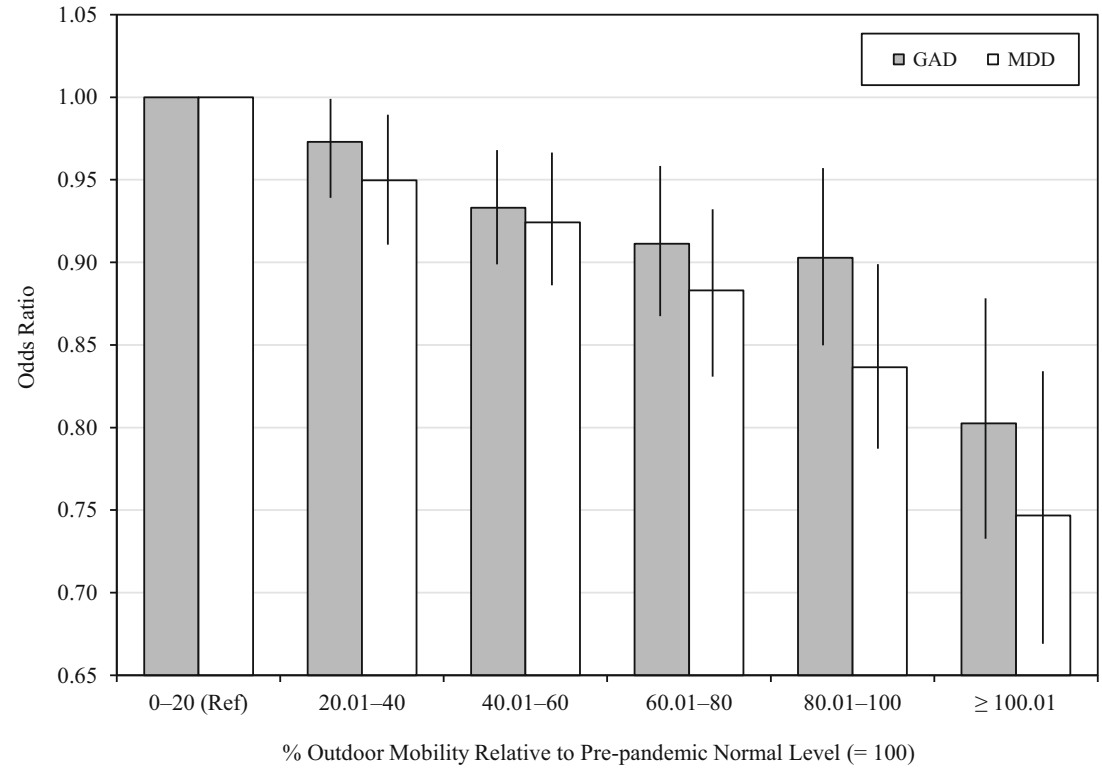


Also, Fig. 2 shows that the highest odds ratio for MDD in states with mobility of $0-20 \%$. Relative to the 0-20\% category, a continued decrease in odds of MDD was found for adults living states with mobility of $20.01-40 \%, 40.01-60 \%, 60.01-80 \%$, and $80.01-$ $100 \%$; beyond $100 \%$, increases in mobility led to the greatest decline in the odds ratio for MDD.

\section{Sensitivity Analyses}

As noted earlier, there are a set of critical assumptions inherent in the analysis that warrant sensitivity analyses and additional evidence on their robustness.

The first test concerns the unique data structure of HPS which is mixture of cross-sectional composition and longitudinal one, as discussed in the data section. While the pooled cross-sectional data allowed us to observe different respondents in different survey weeks, the longitudinal data can deal with repeated responses from the same respondents (identified by scram in HPS microdata) across weeks (maximum 3 weeks). To test if longitudinal data suggest different estimation results than those from the pooled cross-sectional data, we build and run conditional fixed-effects panel logistic regression and random-effects panel logistic regression for GAD and MDD, respectively (see Supplemental Table 3 for the full panel model results). The results for key covariates in the panel models are substantively unchanged, suggesting that both cross-sectional composition and longitudinal composition of HPS data show a consistent relationship between small business closure and reduced urban mobility and mental health outcomes during the COVID-19 pandemic.

Second, there might be some concerns about aggregating different types of small business. This is because a closure of some sectors that serve health care and social assistance may have a detrimental impact on health status of local residents. Also, small stores that provide locations for gathering and chatting may substantially limit socializations and therefore influence mental health outcomes. To partly reflect the sectoral variations, we repeated the main analysis with four SBPS-based covariates that measure weekly closure rate of small businesses in (i) retail (NAICS 2-digit code 44), (ii) health care and social assistance (62), (iii) arts, entertainment, and recreation (71), and (iv) accommodation and food services (72), respectively (see Supplemental Table 4). Note that we use nationwide measure due to unavailability of state-level sectoral data. We find that a greater closure rate of health care stores and social assistance businesses is related with an increased risk of both GAD and MDD across the nation. Estimated coefficients for other sectors were not significant except for the positive association between accommodation and food services closure and MDD, which is contrary to expectations.

Third, we recognize that the specification of a particular state in a particular survey week as locked down and reopened is subject to data source. To test whether an alternative specification might influence the results, we use Raifman et al.'s (2020) COVID19 US State Policy (CUSP) database to determine lockdown and reopening in each week across states (see Supplemental Table 5). The signs and magnitudes of estimated coefficients are quite similar, showing a lower risk of GAD in reopened states while no relations between state reopening and MDD. It suggests that the measure of statewide lockdown and reopening is robust across alternative specifications.

An additional set of sensitivity tests is conducted with regard to alternative dependent and independent variables, different standard errors, and other model specifications. We substituted a comorbidity of GAD and MDD or at least one of the two disorders instead of existing separate measures and found that the model estimations are stable in alternative measures (see Supplemental Table 6). Alternatively, continuous measures of GAD and MDD were tested with the same set of covariates by multilevel mixed effects generalized linear models (meglm in Stata/ MP 13.1), suggesting robust estimation results (see Supplemental Table 7). As key covariates of our interest, small business closures and urban mobility were estimated to have coefficients that are consistent with signs and significance of their quartile measures (see Supplemental Table 8). We also find minimal differences for model estimates when robust standard errors and clustered (clustered by state) standard errors were adopted instead of standard errors (see Supplemental Table 9). Lastly, we estimated weighted logistic models weighted by person-level weight variable (pweight in HPS microdata) for GAD and MDD, respectively, and confirmed that the estimated coefficients in the weighted models were largely unchanged (see Supplemental Table 10). 


\section{Discussion}

COVID-19 and Urban Health Implications

Our findings suggest that social distancing interventions and consequently limited access to socialization locations measured as small business closures and the reduced urban mobility inevitably increased the incidence of anxiety and/or depression among Americans during the COVID-19 pandemic. It is consistent with early findings from other countries such as China, Italy, and Netherland [24-26]. Despite the protective effect of social distancing measures on transmission of the virus, the adverse mental health consequences should be considered from urban health perspectives. As the World Health Organization (WHO) and the UK have issued guidance on the management of mental health aspects of COVID-19 [27, 28], it is necessary to provide a set of individual, community, and state-level interventions for the inevitability of loneliness and its sequelae [3, 29]. Extra efforts should be made to ensure mental health of marginalized populations, such as racial/ethnic minorities, elderly, homeless individuals, and those with serious chronic mental illness [3, 5, 30, 31]. Lastly, existing free and confidential hotlines can be extended to provide immediate mental health supports for vulnerable populations during the pandemic, including children [32], pregnant women [33], health care workers [24, 34], and physicians [35].

An equally important finding is that a wide range of social and economic hardships during the pandemicincluding employment income loss, food insufficiency, and housing payment delay - is closely related with the prevalence of anxiety and depression. These findings may suggest needs for interdisciplinary disaster management plans across different policy arenas. This interconnected policy frame is vital to formulating COVID19 policies and mental health system and also to building the broad citizen support required for any reforms to be successful [36, 37]. Public understanding about these policy interconnections needs to be illuminated and fostered with timely studies on COVID-19 [38].

Strengths, Limitations, and Conclusions

To the best of knowledge, this is the first study on the associations of small business closure and reduced urban mobility with GAD and MDD during COVID-19 pandemic. The national representative sample of HPS data joined with SBPS and smartphone data allowed us to examine the overall picture of adverse mental health outcomes due to reduced socializations.

Our analysis is not without limitations. HPS data are limited in scope, and we were unable to control for a full-range of individual and household-level factors. The contextual and behavioral changes measured from HPS were aggregated to state-level and susceptible to the modifiable areal unit problem (MAUP) [39], which means that the results may change when a different geographic area (e.g., county) other than state is adopted. Our state-level analysis may also suffer from ecological fallacy [40], because the associations from state-aggregated data may not directly translate into associations for individuals who reside in different states. Despite the use of state-level random effects in our models, some state-level covariates (e.g., rurality of each state) may relate to both urban mobility and mental health outcomes, which might lead to over or underestimation of the coefficient of urban mobility. We recognize that the statewide lockdown and reopening were a complicated administrative and policy process, with multiple phases and dimensions to be considered, and therefore our binary specification of state reopening is subject to oversimplification of the reality. Measures of state reopening can be detailed by developing an alternative measure - for example, ordinal measure (e.g., reopening phase of 1,2 , and 3 ) or categorical measure (e.g., reopening of school/daycare, restaurant/bars, sporting venue, and/or place of worship) instead of the binomial form as adopted in this paper - to reflect multiple aspects and varying degree of state reopening policy. Lastly, we did not examine other mental illnesses such as trauma- and stressor-related disorder (TSRD) and seriously considered suicide related to COVID-19. Despite these limitations, this study can shed new light on the role of small business conditions and urban mobility in explaining the increased prevalence of mental illness during COVID-19 pandemic. Further research is needed on longitudinal analysis and individual-level mobility measures, in addition to analysis at lower geographic scales.

Overall conclusion is that, for GAD, both a higher level of small business closures and a lower level of urban mobility were associated with increased prevalence rate of GAD during COVID-19 pandemic. For MDD, however, an increased level of small business closure had no association with the prevalence rate of 
MDD while a reduced level of mobility was strongly associated with increased prevalence of MDD.

Supplementary Information The online version contains supplementary material available at https://doi.org/10.1007/s11524020-00511-0.

Acknowledgements The authors would like to thank anonymous reviewers and the editor-in-cheif for their valuable and constructive suggestions.

Funding The authors received no financial support for the research, authorship, and/or publication of this article.

\section{References}

1. Pfefferbaum B, North CS. Mental health and the Covid-19 pandemic. N Engl J Med. 2020;383(6):510-2.

2. Razai MS, Oakeshott P, Kankam H, Galea S, StokesLampard H. Mitigating the psychological effects of social isolation during the Covid-19 pandemic. BMJ. 2020;369: m1904.

3. Galea S, Merchant RM, Lurie N. The mental health consequences of COVID-19 and physical distancing: the need for prevention and early intervention. JAMA Intern Med. 2020;180:817-8.

4. Galea S. Together in a time of physical distancing. Lancet. 2020;396(10248):371-2.

5. Galea S, Keyes K. Understanding the Covid-19 pandemic through the lens of population health science. Am J Epidemiol. 2020;189:1232-7.

6. Czeisler ME, Lane RI, Petrosky E, Wiley JF, Christensen A, Njai R, et al. Mental health, substance use, and suicidal ideation during the COVID-19 pandemic - United States, June 24-30, 2020. MMWR Morb Mortal Wkly Rep. 2020;69(32):1049-57.

7. Household Pulse Survey. U.S. Census Bureau. 2020. https://www.census.gov/programs-surveys/householdpulse-survey.html.

8. National Health Interview Survey 2019. Center for Disease Control and Prevention. 2020. https://www.cdc. gov/nchs/nhis/index.htm.

9. Rollston R, Galea S. COVID-19 and the social determinants of health. Am J Health Promot. 2020;34(6):687-9.

10. Sinha M, Kumar M, Zeitz L, Collins PY, Kumar S, Fisher S, et al. Towards mental health friendly cities during and after COVID-19. Cities Health. 2020;1-4.

11. Small Business Pulse Survey. U.S. Census Bureau; 2020. https://www.census.gov/data/experimental-dataproducts/small-business-pulse-survey.html.

12. Centers for Disease Control and Prevention (CDC). CDC COVID Data Tracker. Centers for Disease Control and Prevention (CDC); 2020. https://covid.cdc.gov/covid-data- tracker/?utm_source=morning_brew\#cases. Accessed 15 Aug 2020.

13. Warren M, Skillman S. Mobility changes in response to COVID-19. Santa Fe, NM: Descartes Labs; 2020.

14. Household Pulse Survey. U.S. Census Bureau; 2020. https://www.census.gov/data/experimental-dataproducts/household-pulse-survey.html.

15. Manville M, Monkkonen P, Lens M, Green R. COVID-19 and Renter Distress: Evidence from Los Angeles. UCLA Report: UCLA Lewis Center For Regional Policy Studies, USC Lusk Center for Real Estate; 2020.

16. Raudenbush SW, Bryk AS. Hierarchical linear models: Applications and data analysis methods. Vol 1: SAGE; 2002.

17. StataCorp L. Stata multilevel mixed-effects reference manual. College Station, TX: StataCorp LP; 2013.

18. Plummer F, Manea L, Trepel D, McMillan D. Screening for anxiety disorders with the GAD-7 and GAD-2: a systematic review and diagnostic metaanalysis. Gen Hosp Psychiatry. 2016;39:24-31.

19. Arroll B, Goodyear-Smith F, Crengle S, Gunn J, Kerse N, Fishman T, et al. Validation of PHQ-2 and PHQ-9 to screen for major depression in the primary care population. Ann Fam Med. 2010;8(4):348-53.

20. Gupta S, Nguyen TD, Rojas FL, et al. Tracking public and private responses to the COVID-19 epidemic: Evidence from State and Local Government actions. Cambridge, MA: National Bureau of Economic Research Working Paper Series. 2020; No. 27027.

21. Galea S, Abdalla SM. COVID-19 pandemic, unemployment, and civil unrest: underlying deep racial and socioeconomic divides. JAMA. 2020;324:227-8.

22. Wolfson JA, Leung CW, Kullgren JT. Food as a critical social determinant of health among older adults during the coronavirus disease 2019 (COVID-19) Pandemic. JAMA Health Forum. 2020;1(7):e200925.

23. Mehdipanah R. Housing as a determinant of COVID-19 inequities. Am J Public Health. 2020;110:1369-70.

24. Rossi R, Socci V, Pacitti F, di Lorenzo G, di Marco A, Siracusano A, et al. Mental health outcomes among frontline and second-line health care workers during the coronavirus disease 2019 (COVID-19) pandemic in Italy. JAMA Netw Open. 2020;3(5):e2010185.

25. Taquet M, Quoidbach J, Fried EI, Goodwin GM. Mood homeostasis before and during the coronavirus disease 2019 (COVID-19) lockdown among students in the Netherlands. JAMA Psychiatry. 2020.

26. Shi L, Lu Z-A, Que J-Y, Huang XL, Liu L, Ran MS, et al. Prevalence of and risk factors associated with mental health symptoms among the general population in China during the coronavirus disease 2019 pandemic. JAMA Netw Open. 2020;3(7):e2014053.

27. World Health Organization. Mental health policy and service guidance package. Geneva: World Health Organization; 2020.

28. Public Health England. Guidance for the public on the mental health and wellbeing aspects of coronavirus (COVID-19). In: England PH (ed.), UK GOV; 2020.

29. Amsalem D, Dixon LB, Neria Y. The Coronavirus Disease 2019 (COVID-19) outbreak and mental health: Current risks 
and recommended actions. JAMA Psychiatry. 2020. https://doi.org/10.1001/jamapsychiatry.2020.1730.

30. Druss BG. Addressing the COVID-19 pandemic in populations with serious mental illness. JAMA Psychiatry. 2020;77: 891-2.

31. Li Y, Galea S. Racism and the COVID-19 epidemic: recommendations for health care workers. Am J Public Health. 2020;110(7):956-7.

32. Golberstein E, Wen H, Miller BF. Coronavirus disease 2019 (COVID-19) and mental health for children and adolescents. JAMA Pediatr. 2020;174:819-20.

33. Hermann A, Fitelson EM, Bergink V. Meeting maternal mental health needs during the COVID-19 pandemic. JAMA Psychiatry. 2020. https://doi.org/10.1001 /jamapsychiatry.2020.1947.

34. Lai J, Ma S, Wang Y, Cai Z, Hu J, Wei N, et al. Factors associated with mental health outcomes among health care workers exposed to coronavirus disease 2019. JAMA Netw Open. 2020;3(3):e203976.
35. Abbasi J. Prioritizing physician mental health as COVID-19 marches on. JAMA. 2020;323(22):2235-6. https://doi. org/10.1001/jama.2020.5205.

36. Auerbach J, Miller BF. COVID-19 exposes the cracks in our already fragile mental health system. Am J Public Health. 2020;110:969-70.

37. Morabia A. COVID-19: health as a common good. Am J Public Health. 2020;110:1111-2.

38. Edwards JK, Lessler J. What now? Epidemiology in the wake of a pandemic. Am J Epidemiol. 2020. https://doi. org/10.1093/aje/kwaa159.

39. Kwan M-P. The uncertain geographic context problem. Ann Assoc Am Geogr. 2012;102(5):958-68.

40. Schwartz S. The fallacy of the ecological fallacy: the potential misuse of a concept and the consequences. Am J Public Health. 1994;84(5):819-24.

Publisher's Note Springer Nature remains neutral with regard to jurisdictional claims in published maps and institutional affiliations. 